\title{
Estudo Fatorial de um Inventário de Práticas e Crenças Parentais
}

\author{
Selma de Cássia Martinelli - Universidade Estadual de Campinas, Campinas, Brasil \\ Elaine Cristiane Aguena-Matsuoka - Universidade Estadual de Campinas, Campinas, Brasil \\ Débora Cecilio Fernandes - Universidade Estadual do Centro-Oeste (Unicentro, PR), Irati, Brasil
}

\begin{abstract}
Resumo
O objetivo deste estudo foi buscar evidências de validade do Inventário de Práticas e Crenças Parentais (IPCp), mediante análise fatorial exploratória e confirmatória. Foram realizados dois estudos abrangendo um total de 509 participantes, na sua maioria, mães. No primeiro, participaram 247 pais e, no segundo, 262. No primeiro estudo, foi realizada uma análise fatorial exploratória que originou um conjunto final de 15 itens, distribuídos em dois fatores: práticas e crenças parentais. Os coeficientes de alfa de Cronbach foram respectivamente de 0,820 e 0,788 . No segundo estudo, foi realizada uma análise fatorial confirmatória, e os resultados revelaram que o modelo de dois fatores, sugerido no primeiro estudo e agrupados da forma indicada, fornece o melhor ajuste possível para esses dados. Apesar dos bons indicadores psicométricos obtidos, ainda são necessários outros estudos com o inventário.
\end{abstract}

Palavras-chave: relações pais-filho, análise fatorial, família, psicometria

Factorial Study about Parental Practices and Beliefs Inventory

\begin{abstract}
The goal of this study was to seek for evidence of validity for the Parental Practices and Beliefs Inventory (IPCp), using confirmatory and exploratory factor analysis. Two studies were carried out involving 509 participants, mostly mothers,. The first study included 247 parents, and the second, 262. In the first study an exploratory factor analysis was conducted, which originated a final group of 15 items distributed in two factors: parental practices and parental beliefs. The Cronbach's Alpha coefficients were, respectively, 0.820 and 0.788 . In the second study, a confirmatory factor analysis was conducted and the results confirmed that the two-factor model suggested in the first study and since grouped as indicated, provides the best possible fit for these data. Despite the good psychometric indicators obtained, further studies about this inventory are still necessary.

Keywords: Parent-Child Relations; factor analysis; family, psychometrics
\end{abstract}

\section{Estudio Factorial del Inventario de Prácticas y Creencias de los Padres}

\begin{abstract}
Resumen
El objetivo de este estudio fue buscar evidencias de validez en el Inventario de Prácticas y Creencias de los Padres (IPCq), por medio de análisis factorial exploratorio y confirmatorio. Se realizaron dos estudios del cual participaron 509 personas, en su mayoría madres, 247 participantes en el primer grupo y 262 en el segundo. En el primer estudio se realizó un análisis factorial exploratorio que originó un conjunto final de 15 ítems, distribuídos en dos factores: Prácticas y Creencias de los Padres. Los coeficientes Alfa de Cronbach fueron respectivamente, 0,820 y 0,788 . El segundo estudio realizó un análisis factorial confirmatorio y los resultados revelaron que el modelo de dos factores, sugerido en el primer estudio y agrupados de forma indicada, ofrecen el mejor ajuste para esos datos. Pese a los buenos indicadores psicométricos obtenidos, todavía son necesarios otros estudios con el inventario.

Palabras-clave: Relaciones Padre-Hijo; análisis factorial; familia, psicometría
\end{abstract}

Nas últimas décadas, as mudanças vivenciadas pela sociedade têm apontado para uma série de reorganizações e, dentre elas, pode-se destacar a que vem sendo presenciada nas configurações familiares. Essas reorganizações, assim como outras verificadas na sociedade, têm repercutido nos indivíduos e imposto mudanças na forma de pensar e de se relacionar (Dessen, 2010).

De acordo com Dessen e Polonia (2007), a família é a primeira instituição social que, em conjunto com outras, busca promover o bem-estar de seus membros, transmitindo os valores, as crenças e os princípios que estão presentes em uma sociedade. Nesse sentido, a família exerce um impacto significativo e uma forte influência no comportamento do indivíduo, especialmente das crianças, que aprendem as diferentes formas de existir, de ver o mundo e de construir as suas relações sociais.

As dinâmicas familiares propiciam a formação de repertórios comportamentais e de resoluções de problemas que permitem ao indivíduo conhecer e controlar as suas emoções, a expressar os seus sentimentos e a lidar com as diversidades e adversidades da vida. Inicialmente desenvolvidas no âmbito familiar, as formas de expressão dos sentimentos terão repercussão 
em outros ambientes no qual a criança, o adolescente e o adulto interagem (Dessen \& Polonia, 2007; Pratta \& Santos, 2007).

Ao longo do tempo, a estrutura e a dinâmica familiar modificaram-se profundamente e foram identificadas novas características deste grupo, como, por exemplo, a diminuição de famílias numerosas devido à redução do número médio de filhos, o aumento de famílias recompostas em virtude do crescente número de divórcios e, mais recentemente, o aparecimento de famílias homossexuais (Dias, 2011). Essas mudanças, ainda que não totalmente conhecidas, apontam para a necessidade de estudos que busquem conhecer esses novos padrões, já que uma discussão mais extensiva sobre o tema é ainda restrita. Tal necessidade também se impõe tendo em vista que a família, presente em todas as sociedades, é a principal instituição responsável pelos cuidados e estímulos necessários ao crescimento e desenvolvimento da criança.

Conforme apontado na revisão de estudos conduzida por Santos, Martinelli e Monteiro (2012), muitas pesquisas em Psicologia detiveram-se sobre aspectos do contexto familiar. Os autores ainda destacam que há um interesse recorrente nessas investigações. $\mathrm{Na}$ literatura, parte desses estudos tem abordado o suporte familiar (Lemos, Baptista, \& Carneiro, 2010; Ventura \& Noronha, 2014; Camargo, Calais, \& Sartori, 2015), os estilos parentais (Santos, Fônseca, Brasileiro, Andrade, \& Freitas, 2014; Rinhel-Silva, Constantino, \& Rondini, 2012; Faria, Pinto, \& Vieira, 2015), as práticas parentais (Hoffman, 1975; Darling \& Steinberg,1993; Sampaio \& Vieira, 2010; Freitas \& Piccinini, 2010; Bolsoni-Silva \& Loureiro, 2011; Toni \& Hecaveí, 2014) e as crenças parentais (Englund, Luckner, Whaley, \& Byron, 2004; Martinelli \& Aguena, 2011; Santos \& Moreira, 2012).

$O$ presente estudo também tem interesse na temática relativa ao contexto familiar e se deterá, especificamente, sobre as práticas e crenças parentais em relação aos filhos. De acordo com o estudo elaborado por Claes, Lacourse, Bouchard e Perucchini (2003), as práticas parentais podem ser compreendidas pelas estratégias de comunicação, interação, participação e afetividade (emocional bolding), supervisão, controle assertivo (parent supervision) e controle punitivo (punitiveness). Essas estratégias são utilizadas pelos pais como norteadoras para orientar os filhos.

Por sua vez, as crenças parentais podem ser definidas como um conjunto organizado de expectativas que resulta tanto da interação entre pais e filhos quanto da cultura acumulada no grupo social de referência familiar (Goodnow \& Collins, 1990; Molnar, 2000; Santos \& Moreira, 2012). Os pais possuem ideias a respeito de como devem tratar os seus filhos, em função de crenças desenvolvidas sobre o que acreditam ser bom ou ruim. Dessa forma, pode-se considerar que as práticas parentais são influenciadas pelas crenças parentais (Molnar, 2000; Kobarg, Sachetti, \& Vieira, 2006; Santos e Moreira, 2012).

Dretske (1983) acrescenta que a crença se relaciona ao processo de acreditar, sendo considerada como uma forma de expressar algo que se encontra no íntimo do pensamento do sujeito. Assim, entendese que, conforme apontam Santos e Moreira (2012), as crenças parentais estão implícitas nas ações do dia a dia e nas interações entre pais e filhos. Além disso, as crenças dos pais também são consideradas importantes no processo de escolarização dos alunos (Molnar 2000) e no seu bem-estar físico, social e psicológico (Pratta \& Santos, 2007).

Os estudos supracitados indicam a importância de se olhar mais detidamente sobre os diferentes aspectos envolvidos nas relações entre pais e filhos presentes no contexto familiar. À medida que cresce essa preocupação, aumenta também a necessidade de novas investigações que permitam aprofundar os conhecimentos nessa área. Entretanto, há dificuldades em aferição desse contexto, o que tem sido, entre outros motivos, um entrave para o estudo e a realização dessas pesquisas. Ainda que pesem tais dificuldades, instrumentos vêm sendo propostos, na maior parte direcionados aos pais, com o intuito de avaliar os recursos materiais (Marturano,1999), os estilos parentais (Costa, Teixeira, \& Gomes, 2000; Gomide, 2006; Pasquali et al., 2012; Valentini, Alchieri, \& Laros, 2013), o suporte familiar (Baptista, 2009), a qualidade na interação familiar (Weber, Salvador, \& Brandenburg, 2009), práticas de envolvimento parental entre pais e filhos (Teodoro, Benetti, Schuatz, \& Mônego, 2010), as habilidades sociais educativas parentais (Bolsoni-Silva \& Loureiro, 2010), as práticas parentais (Teixeira, Oliveira, \& Wottrich, 2006; Hernández-Guzmán, Montesinos, Bermudez-Ornelas, Anjel-Freire, \& Alcázar-Olán, 2013) e as crenças parentais (Santos \& Moreira, 2012), entre outros.

$\mathrm{Na}$ tentativa de contribuir com uma ferramenta de pesquisa, que possibilite investigar o contexto familiar, o presente estudo apresenta uma proposta de instrumento de avaliação, destinado a pais, cujo interesse incide sobre as práticas e crenças parentais. Nesse instrumento, as práticas parentais são compreendidas por meio das estratégias de comunicação, 
interação, participação e controle assertivo, identificadas pelos pais em seu comportamento com seus filhos. A comunicação entre pais e filhos é estabelecida quando os pais transmitem as mensagens educativas para seus filhos ou quando recebem as mensagens que as crianças expressam, buscando compreender e aceitar opiniões divergentes. Por sua vez, o envolvimento relaciona-se intimamente com a presença dos pais na realização das atividades dos filhos. Refere-se às atitudes e às condutas dos pais durante a supervisão do comportamento dos filhos, com o intuito de conhecer e interagir em suas atividades.

Por outro lado, as crenças parentais são compreendidas, neste estudo, como um conjunto organizado de expectativas acerca da criança, que regulam a motivação para a tomada de decisão e para o modelo comportamental dos pais, na interação com seus filhos (Dretske, 1983; Goodnow \& Collins, 1990; Molnar, 2000).

Tendo em vista as considerações apresentadas neste texto relativas às práticas e crenças dos pais em relação aos filhos, foi elaborado um conjunto inicial de 81 itens como parte do processo de construção e desenvolvimento do Inventário de Práticas e Crenças Parentais. Os itens foram elaborados a partir dos conceitos expostos e com base em dois instrumentos, quais sejam, The home observation for measurement of the environment-HOME (Caldwell \& Bradley, 1984) e o The parent involvement in children's education scale - PICES (Fantuzzo, Tighe, McWayne, Davis, \& Childs, 2003).

A escala HOME avalia o ambiente familiar com base em seis fatores: responsabilidade emocional e verbal, organização do ambiente, envolvimento com os filhos, relação entre mães e filhos, presença de material/jogos adequados e atitudes educativas. Por outro lado, a escala PICES tem o objetivo de avaliar as atitudes dos pais no processo de escolarização dos filhos e é formada por três fatores: suporte para o aprendizado, participação nas atividades escolares e atitudes negativas dos pais.

Assim posto, o objetivo deste estudo foi buscar evidências de validade de um Inventário de Práticas e Crenças Parentais, mediante análise fatorial exploratória e confirmatória. Para atingir esse objetivo, foram realizados dois estudos apresentados a seguir.

\section{Método}

\section{$\underline{1^{\circ} \text { Estudo }}$}

O primeiro estudo teve como objetivo realizar uma avaliação preliminar dos itens do instrumento, a partir da análise fatorial exploratória, visando o refinamento e uma revisão das questões e a possível eliminação daquelas que continham um conteúdo muito similar entre si ou que não colaboravam suficientemente com os fatores encontrados.

\section{Participantes}

A amostra foi constituída por conveniência, tendo em vista que participaram do estudo aquelas escolas e pais que aceitaram colaborar com a pesquisa. Participaram desse primeiro estudo 247 pais de estudantes de ambos os sexos e com idades entre 19 e 61 anos. Os estudantes eram de ambos os sexos e cursavam o $2^{\circ}$ e $4^{\circ}$ anos do ensino fundamental, de três escolas públicas, do estado de São Paulo. Os dados descritivos da amostra são parciais, tendo em vista que nem todos os participantes responderam às questões do questionário informativo. A Tabela 1 ilustra as características da amostra.

\section{Instrumento}

O conjunto de 81 itens, inicialmente construído, apresentava questões afirmativas e negativas, na forma de autorrelato. Como exemplo de questão afirmativa pode-se citar "Eu acredito que meu filho me fala a verdade". Um exemplo de questão negativa era "Eu trabalho o dia todo e não tenho tempo para meu filho". Cada item apresentava três opções de resposta. Para as questões afirmativas, dois pontos eram atribuídos à opção sempre, um ponto para às vezes $\mathrm{e}$ zero pontos para nunca. Essa pontuação teve seu valor invertido para as questões negativas. Quanto maior a pontuação dos pais, maior é a frequência das práticas e crenças declaradas. A pontuação máxima do inventário era de 162 pontos.

\section{Procedimento de Coleta de Dados}

O presente estudo foi realizado após parecer favorável do Comitê de Ética e Pesquisa da Universidade Estadual de Campinas (protocolo n ${ }^{\circ}$ 0842.0.146.00008). Todos os procedimentos éticos de pesquisa foram levados em consideração. Os participantes foram contatados na reunião semestral de pais realizada pelas escolas. Nessa oportunidade, foram apresentados os objetivos do estudo e a observância da livre participação, sem prejuízo aos pais e aos filhos, caso os pais não quisessem ou se sentissem à vontade para participar. Os pais que aceitaram participar responderam ao instrumento na mesma ocasião, na própria escola, em sala reservada, e somente após assinatura de um Termo de 
Tabela 1

Características Sociodemográficas da Amostra - Estudo 1

\begin{tabular}{lcc}
\hline \multirow{2}{*}{ Responsáveis } & $\%$ respondentes & $\%$ não respondentes \\
\cline { 2 - 3 } Idade & mãe $=85 \%$ & $0,4 \%$ \\
& pai $=14,6 \%$ & $23,1 \%$ \\
\hline & $19-29=14,9 \%$ & \\
Estado civil & $30-39=38,5 \%$ & $5,3 \%$ \\
& $50-49=21,0 \%$ & \\
& casado $=65,2 \%$ & \\
\hline & solteiro $=11,7 \%$ & \\
& viúvo $=2,4 \%$ & \\
Escolaridade & divorciados $=6,1 \%$ & \\
& outros $=9,3 \%$ & \\
& EFC $=10,9 \%$ & \\
& EFI $=13 \%$ & \\
& EMC $=38,5 \%$ & $36 \%$ \\
Carga horária de trabalho (semanal) & EMI $=12,1 \%$ & \\
& ESC $=8,1 \%$ & \\
\hline
\end{tabular}

Nota. EFC: ensino fundamental completo, EFI: ensino fundamental incompleto, EMC: ensino médio completo, EMI: ensino médio incompleto, ESC: ensino superior completo e ESI: ensino superior incompleto.

Consentimento Livre e Esclarecido. O tempo médio de aplicação foi de 40 minutos.

\section{$2^{\circ}$ Estudo}

Um segundo estudo foi realizado com o objetivo de proceder a uma análise fatorial confirmatória, que teve por finalidade aceitar ou rejeitar a configuração de dois fatores, obtida na pesquisa anterior.

\section{Participantes}

Participaram desse estudo 262 pais, inseridos em três escolas da rede estadual de ensino público do estado de São Paulo, de ambos os sexos e com idades entre 22 e 64 anos. Os dados descritivos da amostra encontram-se discriminados na Tabela 3.

Quanto aos estudantes, $132(50,4 \%)$ cursavam o terceiro ano e $130(49,6 \%)$ o quinto ano do ensino fundamental de três escolas públicas do Estado de São Paulo. Com relação ao gênero, $130(49,6 \%)$ crianças pertenciam ao sexo masculino e $132(50,4 \%)$ ao sexo feminino e tinham idades entre 7 e 13 anos.

\section{Instrumento}

Inventário de Práticas e Crenças Parentais (IPCp)

Para esse estudo, os participantes responderam ao conjunto de 15 itens resultantes do primeiro estudo. As opções de resposta e as pontuações atribuídas aos itens permaneceram iguais ao primeiro estudo.

\section{Procedimento de Coleta de Dados}

As pesquisadoras fizeram contato com três escolas, que concordaram participar da pesquisa. Foram adotados, nesse momento, os mesmos procedimentos do primeiro estudo. O tempo médio de aplicação dessa coleta foi de 20 minutos.

\section{Resultados}

$\underline{1^{\circ} \text { Estudo }}$

A medida de adequação da amostra, de KaiserMeyer-Olkin $(\mathrm{KMO}=0,673)$, e o teste de esfericidade 
Tabela 2

Características Sociodemográficas da Amostra - Estudo 2

\begin{tabular}{lcc}
\hline & $\%$ respondentes & $\%$ não respondentes \\
\cline { 2 - 3 } Responsáveis & mãe $=3,2 \%$ & $0 \%$ \\
\hline \multirow{3}{*}{ pai $=16,8 \%$} & $20,2 \%$ \\
& $22-29=12,6 \%$ & \\
\hline & $30-39=40,1 \%$ & $4,2 \%$ \\
Estado civil & $50-49=23,3 \%$ & \\
& casado $=63,4 \%$ & \\
& solteiro $=11,8 \%$ & \\
\hline & viúvo $=0,8 \%$ & \\
& divorciados $=9,2 \%$ & \\
Escolaridade & outros $=10,7 \%$ & \\
& EFC $=9,5 \%$ & \\
& EFI $=15,3 \%$ & \\
& EMC $=38,5 \%$ & $36,3 \%$ \\
Carga horária semanal de trabalho & EMI $=9,5 \%$ & \\
& ESC $=8,4 \%$ & \\
\hline
\end{tabular}

Nota. EFC: ensino fundamental completo, EFI: ensino fundamental incompleto, EMC: ensino médio completo, EMI: ensino médio incompleto, ESC: ensino superior completo e ESI: ensino superior incompleto.

de Bartlett $\left(\chi^{2}=6658,749, g l=3240\right.$ e $\left.p<0,001\right)$, da escala de 81 itens, indicaram a possibilidade de extração de mais de um fator. Os dados foram estudados pela análise de componentes principais, rotação Varimax, com eigenvalues igual ou superior a 1,0. Essa análise forneceu 25 fatores, explicando $73,23 \%$ da variância. Analisando o Scree Plot, observou-se a possibilidade de dois fatores.

Considerando que se desejava uma escala bem mais compacta e que também utilizasse os itens com saturações mais altas nos fatores ou, em outros termos, que fossem mais altamente correlacionados entre si, foi realizada uma análise da consistência interna dos itens, por meio do coeficiente de alfa de Cronbach. Utilizou-se como regra de inclusão de itens nos fatores aqueles que tivessem saturação acima de 0,40, conforme sugerido por Hair, Anderson, Tatham e Black (1995). Nessas novas análises, encontrouse uma medida de adequação de Kaiser-Meyer-Olkin $(\mathrm{KMO}=0,825)$ e teste de esfericidade de Bartlett $\left(\chi^{2}=\right.$ 982,709, $g l=105$ e $p<0,001)$, que permitiram chegar a uma solução interpretável de dois componentes, para um conjunto de 15 itens, que explicaram 45,50\% da variância. $\mathrm{Na}$ Tabela 2 , exibe-se a configuração encontrada.

Observa-se na Tabela 2 que, após a eliminação dos itens com saturação inferior a 0,40 , originou-se um conjunto de 15 itens, sendo que seis deles pertencem ao fator 1 e nove, ao fator 2 . Os valores das cargas fatoriais variaram de 0,496 a 0,805 . O coeficiente de consistência interna (alfa de Cronbach) foi de 0,820 para o fator 1, denominado Práticas parentais. Para o fator 2, denominado de Crenças parentais, o coeficiente de consistência interna foi de 0,788 .

\section{$\underline{2^{\circ} \text { Estudo }}$}

A análise fatorial confirmatória foi realizada com o software Mplus. Esse procedimento de análise exploratório-confirmatória é uma prática comum no desenvolvimento e estudo de instrumentos de avaliação, pois a análise confirmatória posterior à exploratória permite aventar hipóteses a priori sobre a quantidade 
Tabela 3

Saturação (Superior a 0,40) por Componentes Principais, Rotação Varimax e Normatização de Kaiser, Eigenvalues e Variância Explicada

\begin{tabular}{lc}
\hline & Componentes \\
\cline { 2 - 2 } & 1 \\
\hline 1F1. Eu trabalho muito e não consigo acompanhar o que meu filho faz na escola. & 0,805 \\
2F1. Eu não consigo acompanhar a rotina diária do meu filho. & 0,749 \\
3F1. Eu trabalho o dia todo e não tenho tempo para meu filho. & 0,746 \\
4F1. Eu não tenho tempo para conversar com meu filho, pois tenho muitas coisas para fazer. & 0,738 \\
5F1. Eu fico com meu filho apenas os finais de semana. & 0,701 \\
6F1. Eu não participo da vida escolar de meu filho. & 0,620 \\
7F2. Eu acredito que meu filho é sincero com os outros. & 0,683 \\
8F2. Eu acho que meu filho é responsável. & 0,679 \\
9F2. Eu confio em meu filho. & 0,659 \\
10F2. Eu acredito que meu filho me fala a verdade. & 0,652 \\
11F2. Eu acho que meu filho é um bom filho. & 0,629 \\
12F2. Eu acredito que meu filho está indo bem na escola. & 0,597 \\
13F2. Eu acredito que meu filho respeita as pessoas. & 0,582 \\
14F2. Eu acho que meu filho é um bom aluno. & 0,540 \\
15F2. Eu acredito que meu filho é querido pelos seus amigos. & 0,496 \\
\hline Eigenvalues & 4,14 \\
\hline Variância explicada & 27,64 \\
\hline
\end{tabular}

de fatores subjacentes ao instrumento e testar essas hipóteses (Pérez-Gil, Moscoso, \& Rodríguez, 2000).

Foram utilizadas as instruções e recomendações propostas por Albright e Park (2009). Os autores propõem o uso de mais de um indicador de ajuste devido aos problemas e restrições que cada um deles possui. Dessa forma, foram usados os indicadores qui-quadrado, Root Mean Square Error of Approximation (RMSEA), Comparative Fit Index (CFI) e Tucker-Lewis Index (TLI). O estimador dos parâmetros utilizado foi o Weighted Least Squares Means and Variance Adjusted (WLSMV).

O valor do qui-quadrado foi 145,108, com grau de liberdade de 89 e $p=0,0002$. O RMSEA foi de 0,049, $\operatorname{com} p=0,526$. Por sua vez, o CFI foi 0,973 e o TLI foi 0,968 . O qui-quadrado e o valor de $p$ indicaram que os valores encontrados foram suficientemente elevados para rejeitar a hipótese nula de um ajuste adequado. No entanto, esse indicador pode ser problemático devido a sua sensibilidade ao tamanho da amostra e, consequentemente, conforme aumenta o número de casos, esse indicador tem maior dificuldade em aceitar a hipótese nula. Dessa forma, ele não pode ser usado individualmente (Albright \& Park, 2009).

Nesse caso, recomenda-se o uso do RMSEA, CFI e TLI (Albright \& Park, 2009). Diferentemente do qui-quadrado, esses indicadores de ajuste mostraram valores de bom ajuste ao modelo de dois fatores. $\mathrm{O}$ RMSEA foi baixo e não significativo, aceitando, dessa forma, a hipótese nula de um bom ajuste. Os valores de CFI e TLI estão próximos de 1, o que também indica bom ajuste dos dados ao modelo de dois fatores. Assim, considera-se, de forma geral, que os indicadores sugerem que há um bom ajuste dos dados ao modelo proposto de dois fatores.

A seguir, a Tabela 4 exibe os valores da estimação fatorial padronizada dos itens do fator prática parental, $\mathrm{o}$ valor de $p$ e as informações relativas ao $\mathrm{R}$ quadrado. Os valores de $\mathrm{R}$ quadrado indicam quanta variância dos itens é explicada pelos próprios fatores.

Observa-se que todos os itens colaboram significativamente com esse fator e apresentam cargas fatoriais elevadas. Os itens que mais colaboram com 
o fator são o I10, I5 e o I7. Os fatores, por sua vez, explicam significativamente a variância observada nos itens. Como exemplo, o R quadrado indica que o fator práticas parentais explica $66 \%$ da variância total do item I5. A Tabela 5 exibe os dados relativos ao fator de crenças parentais.

A Tabela 5 indica que todos os itens apresentam cargas fatoriais significativas e elevadas, mas não tão alto quanto no fator Práticas. Os itens que mais contribuem com o fator são o I8, I3 e I1. Os valores de R quadrado foram significativos e parte considerável de sua variância é explicada pelos fatores. Por exemplo, o fator Crença explica $54 \%$ da variância total do item I1. A porcentagem explicada de variância mais baixa foi a do item 15, com $30 \%$ explicado pelo fator Crença.
Foi realizada também a análise de correlação entre os fatores Práticas e Crenças. Obteve-se um $r=0,062$ e $p=0,352$, o que significa que não há correlação significativa entre ambos os fatores. Finalmente, foi realizada a análise de melhora de ajuste na condição de eliminação ou troca de fator de algum item. O resultado dessa análise mostrou que não foram sugeridas melhorias ao modelo proposto, quer dizer, o modelo de dois fatores cujos itens estão agrupados dessa forma fornece o melhor ajuste possível para esses dados.

\section{Discussão}

A família talvez seja uma das instituições sociais que mais sofreu alterações nas últimas décadas. Esse

Tabela 4

Estimação Fatorial o Fator Prática Parental

\begin{tabular}{lccccc}
\hline \multicolumn{5}{c}{ Prática parental } \\
\hline \multirow{2}{*}{ Itens } & Estimação & $p$ & \multicolumn{3}{c}{ R quadrado } \\
\cline { 3 - 5 } & & & Estimação & $p$ & Variância residual \\
I5 & 0,813 & 0,000 & 0,661 & 0,000 & 0,339 \\
I7 & 0,825 & 0,000 & 0,681 & 0,000 & 0,319 \\
I10 & 0,833 & 0,000 & 0,695 & 0,000 & 0,305 \\
I11 & 0,766 & 0,000 & 0,587 & 0,000 & 0,413 \\
I12 & 0,690 & 0,000 & 0,476 & 0,000 & 0,524 \\
I14 & 0,622 & 0,000 & 0,387 & 0,000 & 0,613 \\
\hline
\end{tabular}

Tabela 5

Resultados do Modelo para o Fator Crenças Parentais

\begin{tabular}{lccccc}
\hline \multicolumn{5}{c}{ Crenças parentais } \\
\cline { 4 - 6 } Itens & Estimação & $p$ & \multicolumn{3}{c}{ R quadrado } \\
\hline I1 & 0,740 & 0,000 & 0,548 & 0,000 & Variância residual \\
I2 & 0,643 & 0,000 & 0,414 & 0,000 & 0,452 \\
I3 & 0,783 & 0,000 & 0,613 & 0,000 & 0,586 \\
I4 & 0,731 & 0,000 & 0,534 & 0,000 & 0,387 \\
I6 & 0,729 & 0,000 & 0,532 & 0,000 & 0,466 \\
I8 & 0,799 & 0,000 & 0,638 & 0,000 & 0,468 \\
I9 & 0,646 & 0,000 & 0,418 & 0,000 & 0,362 \\
I13 & 0,596 & 0,000 & 0,355 & 0,000 & 0,582 \\
I15 & 0,551 & 0,000 & 0,303 & 0,000 & 0,645 \\
\hline
\end{tabular}


fato talvez seja um dos elementos motivadores que tem instigado pesquisadores a buscarem novas formas de olhar para essas configurações. Investigações também têm sido propostas com o intuito de conhecer as variáveis contextuais, presentes nessas novas formas de organização familiar, e que possam interferir diretamente sobre o aprendizado infantil. Muitas dessas variáveis contextuais têm interessado os pesquisadores, conforme citado na introdução deste estudo, embora ainda se tenha uma diversidade muito pequena de recursos instrumentais para essas investigações.

Partindo dessa lacuna verificada na literatura, o presente estudo teve por objetivo propor um novo inventário que avaliasse aspectos relativos à percepção dos pais sobre suas práticas e crenças em relação aos seus filhos. Como parte do processo de construção e desenvolvimento desse inventário, foi proposto 81 itens. A análise fatorial exploratória desses itens indicou que o melhor ajuste se referia a um conjunto de 15 itens distribuídos em dois fatores, que foram interpretados como de práticas (fator 1) e crenças parentais (fator 2). As análises preliminares do instrumento revelaram qualidades psicométricas bastante satisfatórias, como os valores de alfa, que indicaram que ambos os fatores apresentam bons índices de confiabilidade (Urbina, 2007).

Os itens do fator 1, denominado de Práticas parentais, estão relacionados com o acompanhamento da rotina geral e escolar, da participação na vida do filho e do tempo disponível para estar e conversar com o filho. Considerando que as práticas, segundo Claes et al. (2003), envolvem uma série de estratégias utilizadas pelos pais, dentre elas as que tratam da interação e participação destes na rotina dos filhos, pode-se afirmar que o fator 1 corresponde ao constructo investigado.

Por outro lado, os itens do fator 2 , que apresentaram carga fatorial suficiente para permanecer no instrumento, foram interpretados como sendo de crenças parentais relacionadas ao contexto escolar e conduta social. Os itens referem-se a crenças positivas dos pais e que se encontram relacionadas à confiança e a expectativa que nutrem no potencial dos filhos, como acreditar que o filho é responsável, que é um bom filho, um bom aluno, confiar nele, acreditar que ele diz a verdade, que é querido pelos amigos. Considerando que as práticas de cuidado e de educação dos pais podem ser influenciadas pelas crenças parentais, é possível considerar que os pais que confiam e possuem expectativas positivas com relação ao potencial dos seus filhos poderão desenvolver atitudes mais positivas de apoio a eles.
Análises complementares a esse primeiro estudo foram conduzidas com a finalidade de verificar a adequação dessa versão de dois fatores, bem como a relação entre eles. Para atingir a esse objetivo foi realizado um segundo estudo para proceder a uma análise fatorial confirmatória (AFC). Os resultados da AFC indicaram que a melhor explicação para o conjunto de 15 itens resultantes da análise anterior é por meio de dois fatores, já que os indicadores de ajuste, com exceção do qui-quadrado, não rejeitaram a hipótese de ajuste de um modelo de dois fatores. Nesse contexto, a literatura recomenda não usar apenas o qui-quadrado, devido aos problemas já revelados em outros estudos (Albright \& Park, 2009). É indicado também o uso de mais de um indicador de ajuste, pois todos eles possuem suas limitações. No caso deste estudo, como houve ajuste pelos indicadores RMSEA, CFI e TLI, pode-se interpretar a adequação da configuração dos itens em dois fatores. Assim, a AFC corroborou o resultado da análise fatorial exploratória e reforçou a manutenção dos fatores, práticas e crenças parentais.

Um resultado interessante, que merece menção, foi fornecido pelo programa Mplus, e refere-se à quantidade de variância dos itens explicada por seus respectivos fatores. Esse dado acrescenta informações sobre a adequabilidade da configuração do inventário dividido em dois fatores, pois ajuda a justificar a permanência dos itens em cada fator. Isso quer dizer que se um item apresenta uma quantidade baixa de variância explicada pelo fator, talvez esse item não seja adequado para esse fator e sua variância seja explicada melhor por outro fator. No caso do referido instrumento, e seus dois fatores, essa análise permitiu justificar a permanência dos itens em cada fator. É interessante destacar, ainda, que todos os itens possuem cargas fatoriais muito altas, o que indica que colaboram muito para seus respectivos fatores.

Nesse sentido, tais resultados reforçam a noção de que o conteúdo dos itens do fator relacionado às práticas refere-se às estratégias de comunicação, interação e participação dos pais em relação aos filhos, conforme apontado no estudo de Claes et al. (2003). Por sua vez, os itens do fator relativo às crenças parentais dizem respeito às situações, como aponta Dretske (1983), que envolvem o acreditar na conduta social e ter expectativas positivas, presentes ou futuras, relativas ao processo de escolarização da criança.

Embora os itens dos dois fatores representem elementos dos constructos propostos, qual seja, o de práticas e de crenças parentais, e que haja na literatura 
indicações teóricas (Molnar, 2000; Kobarg, et al., 2006; Pacheco \& Mata, 2013), que apontem que as crenças se constituem como referencial importante para determinar certas atitudes ou práticas parentais, os resultados deste estudo indicaram que, para esse conjunto de itens, não houve correlação entre os dois fatores. Nesse caso, as ações representadas pelo fator de práticas não se correlacionaram com as crenças dos pais em relação aos seus filhos, o que indica tratar-se de dois instrumentos independentes. Nesse sentido, considera-se interessante continuar investigando se os dois fatores encontrados devem fazer parte de um mesmo instrumento ou serem tratados como dois instrumentos diferentes, uma vez que não se encontrou estudos empíricos que tenham analisado a relação entre estes dois constructos da forma como foi proposto neste estudo.

Assim, ainda que o instrumento revele propriedades psicométricas bastante adequadas, é importante ainda ressaltar que, seria oportuna a realização de outros estudos em que fossem contempladas uma ampliação da população amostral e uma maior diversidade dos participantes. Considera-se também a possibilidade de desenvolver novos itens que fizessem referência a uma diversidade maior de situações relacionadas às práticas parentais, já que, aparentemente, o conteúdo dos itens não revela toda a abrangência possível do constructo. Igualmente importante seria rever as opções de resposta dos itens, quer dizer, realizar estudos que indiquem se os itens funcionam bem com três opções de resposta ou se um padrão dicotômico seria mais adequado.

\section{Referências}

Albright, J. J., \& Park, H. M. (2009). Confirmatory factor analysis using Amos, LISREL, Mplus, SAS/ STAT CALIS. Working paper. The University Information Technology Services (UITS). Center for Statistical and Mathematical Computing. Indiana University. Recuperado de http://www.indiana. edu/ statmath/stat/all/cfa/cfa.pdf

Baptista, M. N. (2009). Inventário de percepsão de suporte familiar-IPSF. São Paulo: Vetor.

Barreto, M. J., \& Rabelo, A. A. (2015). A família e o papel desafiador dos pais de adolescentes na contemporaneidade. Pensando Famílias, 19(2), 34-42. Recuperado de http://pepsic.bvsalud.org/pdf/ penf/v19n2/v19n2a04.pdf
Bolsoni-Silva, A. T., \& Loureiro, S. R. (2011). Práticas educativas parentais e repertório comportamental infantil: Comparando crianças diferenciadas pelo comportamento. Paidéia, 21(48), 61-71. doi: 10.1590/S0103-863X2011000100008

Bolsoni-Silva, A. T., \& Loureiro, S. R. (2010). Validação do roteiro de entrevista de habilidades sociais educativas parentais (RE-HSE-P). Avaliação Psicológica, 9(1), 63-75. Recuperado de http://www.redalyc. org/pdf/3350/335027281008.pdf

Cadwell, B. \& Bradley, R. (1984). Home Observation for Measurement of the Environment. Little Rock, AR: University of Arkansas.

Camargo, V. C. V., Calais, S. L., \& Sartori, M. M. P. (2015). Estresse, depressão e percepção de suporte familiar em estudantes de educação profissionalizante. Estudos em Psicologia, 32(4), 595-604. doi: 10.1590/0103-166X2015000400003

Claes, M., Lacourse, E., Bouchard, C., \& Perucchini, P. (2003). Parental practices in late adolescence, a comparison of three countries: Canada, France and Italy. Journal of Adolescence, 26(4), 387-399. Recuperado de http://www.mapageweb.umontreal. ca/lacoure/doc/Claes_2003_JA.pdf

Costa, F. T., Teixeira, M. A., \& Gomes, W. B. (2000). Responsividade e exigência: Duas escalas para avaliar estilos parentais. Psicologia: Reflexão e Crítica, 13(3), 465-473. doi: 10.1590/S0102-79722000000300014

Darling, N., \& Steinberg, L. (1993). Parenting style as context: An integrative model. Psychological Bulletin, 113(3), 487-496. doi: 10.1037/0033-2909.113.3.487

Dessen, M. A., \& Polonia, A. D. C. (2007). A família e a escola como contextos de desenvolvimento humano. Paidéia, 17(36), 21-32. doi: 10.1590/S0103-863X2007000100003

Dessen, M. A. (2010). Estudando a família em desenvolvimento: Desafios conceituais e teóricos. Psicologia Ciência e Profissão, 30(spe), 202-219. Recuperado de http://www.scielo.br/pdf/pcp/v30nspe/v30speca10.pdf

Dias, M. O. (2011). Um olhar sobre a família na perspectiva sistêmica - O processo de comunicação no sistema familiar. Gestão e Desenvolvimento, 19(1), 139-156. Recuperado de http://repositorio.ucp. pt/bitstream/10400.14/9176/1/gestaodesenvolvimento19_139.pdf 
Dretske. F. I. (1983). The epistemology of beliefs. Synthese. 55(1), 3-19. doi: 10.1007/BF00485371

Englund, M. M., Luckner, A. E., Whaley, G., \& Byron, E. (2004). Children`s achievement in early elementary school: Longitudinal effects of parental involvement expectations, and quality of assistance. Journal of Education Psychology, 96(4), 723-730. doi: 10.1037/0022-0663.96.4.723

Fantuzzo, J., Tighe, E., McWayne, C., Davis, G., \& Childs, S. (2003). Parent involvement in early childhood education and children's peer-play competencies: An examination of multivariate relationshiops. NHS A Dialog, 6(1), 3-21. doi: 10.1207/ s19309325nhsa0601_2

Faria, L. C., Pinto, J. C., \& Vieira, M. (2015). Construção da carreira: O papel da percepção dos filhos acerca dos estilos educativos parentais na exploração vocacional. Psicologia: Reflexão e Crítica, 28(1), 194-203. doi: 10.1590/1678-7153.201528121

Freitas, A. P. C. O., \& Piccinini, C. A. (2010). Práticas educativas parentais em relação ao filho único e primogênito. Estudos em Psicologia, 27(4), 515-528. doi: 10.1590/S0103-166X2010000400009

Gomide, P. I. C. (2006). Inventário de estilos parentais: Modelo teórico, manual de aplicação, apuração e interpretação. Petrópolis: Vozes.

Goodnow, J. J., \& Collins, W. A. (1990). Development according to parents: The nature, sources, and consequences of parents' ideas. Hillsdale, NJ: Lawrence Erlbaum.

Hair, J. F., Anderson, R. E., Tatham, R. L., \& Black, W. C. (1995). Multivariate data analysis. 4. Ed. New Jersey: Prentice Hall.

Hernández-Guzmán, L., Montesinos, M. G., Bermudez-Ornelas, G., Anjel-Freyre, M., \& Alcázar-Olán, R. (2013). Parental pratices scale for children. Revista Colombiana de Psicologia, 22(1), 151-161. Recuperado de http://www.redalyc.org/ pdf/804/80428081011.pdf

Hoffman, M. L. (1975). Moral, internalization, parental power, and the nature of parent child interaction. Developmental Psychology, 11(2), 228-239. doi: $10.1037 /$ h0076463

Kobarg, A. P. R., Sachetti, A. R., \& Vieira, M. L. (2006). Valores e crenças parentais: Reflexões teóricas. Revista Brasileira de Crescimento e Desenvolvimento Humano,
16(2), 92-102. Recuperado de http://pepsic.bvsalud.org/pdf/rbcdh/v16n2/10.pdf

Lemos, V. A., Baptista, M. N., \& Carneiro, A. M. (2011). Suporte familiar, crenças irracionais e sintomatologia depressiva em estudantes universitários. Psicologia Ciência e Profissão, 31(1), 20-29. Recuperado de http://www.scielo.br/pdf/pcp/v31n1/ v31n1a03.pdf

Martinelli, S., \& Aguena, E. C. (2011). La motivación en estudiantes de enseñanza fundamental y las creencias y actitudes de los padres. Revista de Investigação em Psicologia, 14(1), 53-64. Recuperado de http:// sisbib.unmsm.edu.pe/bvrevistas/investigacion_ psicologia/v14_n1/pdf/a04.pdf

Marturano, E. M. (1999). Recursos no ambiente familiar e dificuldades de aprendizagem na escola. Pesquisa: Teoria e Pesquisa, 15(2), 135-142. Recuperado de http://www.scielo.br/pdf/ptp/v15n2/ a06v15n2.pdf

Molnar, Z. (2000). Parents beliefs about school, attributions about performance, and their children's academic achievement. Magyar-Ps₹ichologiai-Szemle, 55(2-3), 207- 227. doi: 10.1556/ MPSzle.55.2000.2-3.3

Pasquali, L., Gouveia, V. V., Santos, W. S., Fonsêca, P. N., Andrade, J. M., \& Lima, T. J. S. (2012). Questionário de percepção dos pais: Evidências de uma medida de estilos parentais. Paidéia, 22(52), 155164. doi: 10.1590/S0103-863X2012000200002

Pérez-Gil, J. A., Moscoso, S. C., \& Rodríguez, R. M. (2000). Validez de constructo: El uso de análisis factorial exploratorio-confirmatorio para obtener evidencias de validez. Psicothema, 12(2), 442-446. Recuperado de http://www.psicothema.com/ pdf/601.pdf

Pacheco, P., \& Mata, L.(2013). Literacia familiar - Crenças dos pais de crianças em idade pré-escolar e características das práticas de literacia na família. Análise Psicológica, 31(3), 217-234. doi: 10.14417/ ap.722

Pratta, E. M. M., \& Santos, M. A. (2007). Família e adolescência: A influência do contexto familiar no desenvolvimento psicológico de seus membros. Psicologia em estudo, 12(2), 247-256. doi: 10.1590/ S1413-73722007000200005 
Rinhel-Silva, C. M., Constantino, E. P., \& Rondini, C. A. (2012). Família, adolescência e estilos parentais. Estudos de Psicologia, 29(2), 221-230. doi: 10.1590/ S0103-166X2012000200008

Sampaio, I. T. A., \& Vieira, M. L. (2010). A influência do gênero e ordem de nascimento sobre as práticas educativas parentais. Psicologia Reflexão e Crítica, 23(2), 198-207. doi: 10.1590/S0102-79722010000200002

Santos, A. A. A., Martinelli, S. C., \& Monteiro, R. M. (2012). Suportes e recursos familiares: Relações com o contexto escolar. Em M. N. Baptista e M. L. M. Teodoro (Eds.). Psicologia de Família; teoria, avaliação e intervenção. (pp.137-144). Porto Alegre: ARTMED.

Santos, J. L. F., Fonsêca, P. N., Brasileiro, T. C., Andrade, P. O., \& Freitas, N. B. C. (2014). A Relação entre os estilos parentais e o engajamento escolar. Temas em Psicologia, 22(4), 759-769. doi: 10.9788/ TP2014.4-07

Santos, M. P., \& Moreira, P. A. (2012). Desenvolvimento e validação do questionário de avaliação das crenças parentais acerca dos fatores determinantes do rendimento académico dos seus filhos. Journal of Child and Adolescent Psychology, 3(1), 15-38. Recuperado de http://revistas.lis.ulusiada.pt/index. $\mathrm{php} / \mathrm{rpca} /$ article/view/38/pdf

Teixeira, M. A. P., Oliveira, A. M., \& Wottrich, S. H. (2006). Escalas de práticas parentais (EPP): Avaliando dimensões de práticas parentais em relação a adolescentes. Psicologia: Reflexão e Crítica, 19(3), 433-441. doi: 10.1590/s0102-79722006000300012
Teodoro, M. L. M., Benetti, S. P. C., Schwartz, C. B., \& Monêgo, B. G. (2010). Propriedades psicométricas do parental bolding instrument e associação com funcionamento familiar. Avaliação Psicológica, 9(2), 243-251. Recuperado de http://www.redalyc.org/ pdf/3350/335027283009.pdf

Toni, C. G. D. S., \& Hecaveí, V. A. (2014). Relações entre práticas educativas parentais e rendimento acadêmico em crianças. Psico-USF, 19(3), 511-521 doi: 10.1590/1413-82712014019003

Urbina, S. (2007). Fundamentos da testagem psicológica. Porto Alegre: Artmed.

Valentini, F., Alchieri, J. C., \& Laros, J. A. (2013). Evidências de validade da versão reduzida do inventário de estilos parentais de Young. Paidéia, 23(56), 293 300. doi: 10.1590/1982-43272356201303

Ventura, C. D., \& Noronha, A. P. P. (2014). Autoeficácia para escolha profissional, suporte familiar e estilos parentais em adolescentes. Avaliação Psicológica, 13(3), 317-324. Recuperado de http://pepsic.bvsalud.org/pdf/avp/v13n3/v13n3a03.pdf

Weber, L. N. D., Salvador, A. P. V., \& Brandenburg, O. J. (2009). Escalas de qualidade na interação familiar. Em L. Weber \& M. A. Dessen (Eds.), Pesquisando a família. Instrumento para coleta e análise de dados (pp. 57-68). Curitiba: Juruá.

Recebido em: 29/02/2016

Reformulado em: 11/05/2016 Aceito em: 24/05/2016 
Sobre as autoras:

Selma de Cássia Martinelli é graduada em Pedagogia pela Universidade Federal de São Carlos (1987), mestre (1992) e doutora (1998) em Educação pela Universidade Estadual de Campinas (UNICAMP), professora livredocente no Departamento de Psicologia Educacional da Faculdade de Educação da UNICAMP, coordenadora e Líder no CNPq do Grupo de Estudos e Pesquisa em Psicopedagogia (GEPESP) e bolsista de produtividade em Pesquisa do CNPq - Nível 2.

E-mail:selmacm@unicamp.br

Elaine Cristiane Aguena-Matsuoka é graduada em Pedagogia pela Universidade Júlio de Mesquita, mestre (2010) e doutora (2017) em Educação, pela Universidade Estadual de Campinas (UNICAMP).

E-mail: lainenek@hotmail.com

Débora Cecilio Fernandes é graduada em Psicologia pela Universidade Estadual de Londrina e Doutorado em Neuropsicologia clínica pela Universidad de Salamanca (2011), Espanha, com equivalência na área de Neurologia, pela Faculdade de Ciências Médicas da UNICAMP. Atualmente, trabalha como professora colaboradora na Universidade Estadual do Centro-Oeste (Unicentro, PR), no Departamento de Pedagogia e membro do Grupo de Estudos e Pesquisa em Psicopedagogia da UNICAMP (GEPESP).

E-mail: debora.cecilio@gmail.com

Contato com as autoras:

Av. Bertrand Russel, 801

Cidade Universitária Zeferino Vaz

Campinas-SP, Brasil

CEP: 13083-865 